\title{
Always better together: the Canadian Journal of Public Health and the Canadian Society for Epidemiology and Biostatistics
}

\section{Toujours meilleures ensemble : la Revue canadienne de santé publique et la Société canadienne d'épidémiologie et de biostatistique}

\author{
Paul J. Villeneuve ${ }^{1} \cdot$ Gilles Paradis $^{2}$ - Nazeem Muhajarine ${ }^{3}$ \\ Published online: 23 June 2020 \\ (C) The Canadian Public Health Association 2020
}

As the world is grappling with the challenges of navigating the global COVID-19 pandemic, there is a heightened recognition that the discipline of epidemiology is a critical component of public health. Canada's epidemiologists are providing essential input on disease modelling, study design, sampling, surveillance, analysis, risk communications, and the identification of vulnerable populations. The mission of the Canadian Society for Epidemiology and Biostatistics (CSEB) is to promote the interrelated sciences of epidemiology and biostatistics to improve health and well-being through research and practice. Hence, CSEB shares an important part of the mission of the Canadian Public Health Association and our two organizations have a long history of successful collaborations and partnership, including special sections in the Canadian Journal of Public Health (CJPH) to publish research presented at CSEB's biannual conferences. The current special section (Issue 3, June 2020) includes articles that describe some of the research presented at the CSEB conference that was

Paul J. Villeneuve

PaulVilleneuve@CUNET.CARLETON.CA

Gilles Paradis

gilles.paradis@mcgill.ca

Nazeem Muhajarine

nazeem.muhajarine@usask.ca

Carleton University, Ottawa, ON, Canada

McGill University, Montreal, QC, Canada

3 University of Saskatchewan, Saskatoon, SK, Canada held at Carleton University in Ottawa, Ontario, from May 13-15, 2019, under the theme "Epidemiology and Biostatistics at the Nexus of Complex Health Challenges". Attendees were invited to submit manuscripts based on the work they presented at the Conference. These were then submitted to the usual CJPH peer-review mechanism.

The six papers published in this Special Section cover a wide range of topics, and capture an important selection of current Canadian epidemiological research. They address methodological issues, etiological research among infants and aging populations, social determinants of health, and analyses of administrative health data.

Bauer et al. (2020) raise important concerns about the wording that current Canadian surveys use to classify visible minorities, and highlight how these questions do not reliably capture those who are racialized as persons of colour. They seek to understand how Canadians who come from diverse backgrounds interpret the terms "person of colour" and "visible minority", and provide recommendations for how future surveys can better capture racialization. This work provides some empirical evidence to guide researchers on how to incorporate equity, diversity, and inclusivity considerations into their studies.

Duchaine et al. (2020) provide an elegant analysis of data from the Canadian Longitudinal Study on Aging to investigate associations between vitamin $\mathrm{D}$ and the development of dementia. The study makes use of plasma-based measures of vitamin $\mathrm{D}$ collected from some participants within the prospective cohort. Their findings underscore the importance of looking at differential associations between men and women. The authors highlight a need to further pursue investigating these associations, particularly in women, while accounting for survival bias. 
On the other side of the age spectrum, Hu et al. (2020) examine associations between prenatal urinary phthalate metabolite concentrations and preterm birth. These analyses were done using data collected from nearly 2000 women enrolled in the MIREC study whose urinary concentrations of phthalate metabolites were measured during their first trimester. Although, for the most part, no associations between phthalate exposure and preterm birth were found, non-linear trends were suggestive of a non-monotonic dose-response pattern, particularly for male infants. The authors suggest a need to undertake further research with multiple measures of phthalate.

Norwood et al.'s (2020) paper seeks to estimate and map small area measures of the social determinants of health using a Bayesian model that accounts for spatial correlations in the data and provides measures of uncertainty. Their approach overcomes some of the limitations that accompany the use of factor analysis, which to date has been the most commonly used method to create Canadian measures of marginalization. The authors propose extending their approach by including multi-level Bayesian models that incorporate both individual-level and contextual area-level data.

Rosella et al.'s (2020) study estimates, on a regional basis, the extent to which immigration status contributed to variations in premature mortality in Ontario between 1992 and 2012. Their results suggest that "healthy-immigrant" effects are important contributors to spatial variability in mortality. Additional efforts that can identify these impacts at a higher spatial resolution represent an important future research direction, as would the capability to distinguish families by more refined immigration landing categories.

Sandhu et al. (2020) present a secondary review of a series of non-randomized intervention studies designed to prevent obesity in children and youth. While these interventions that were reviewed demonstrate considerable promise, most do not adequately account for clustering, do not apply state-of-the-art causal epidemiological methods, and generally fail to assess effect modification. As such, these original studies are likely presenting biased measures of association, or overstating the precision of their measures of association.

Taken together, these papers illustrate the dynamic nature of epidemiological research in Canada and its important contributions to improving our understanding of public health. They further emphasize the importance of methodological rigour and the application of sound epidemiological principles for the design of population research and analysis of health data. Finally, they reinforce the need for close collaborations and partnerships between the Canadian epidemiological, biostatistical, and public health communities in our unrelenting work to improve the health of all Canadians.

Paul Villeneuve, Gilles Paradis, Nazeem Muhajarine Co-editors, Special Section on Epidemiology and Biostatistics

\section{Éditorial}

Depuis que le monde est aux prises avec la pandémie de COVID-19, le rôle indispensable de la discipline de l'épidémiologie en santé publique est mieux reconnu. Les épidémiologistes du Canada apportent une contribution essentielle à la modélisation des maladies, aux protocoles d'études, à l'échantillonnage, à la surveillance, à l'analyse, aux communications relatives aux risques et à l'identification des populations vulnérables. La Société canadienne d'épidémiologie et de biostatistique (SCEB) a pour mission de promouvoir les sciences apparentées que sont l'épidémiologie et la biostatistique pour améliorer la santé et le bien-être par la recherche et la pratique. Elle partage donc en partie la mission de l'Association canadienne de santé publique, et les deux organismes ont une longue histoire de collaborations et de partenariats fructueux, notamment dans le cadre de rubriques spéciales de la Revue canadienne de santé publique (RCSP) où sont publiées des études de recherche présentées lors du congrès biennal de la SCEB. Les articles de la rubrique spéciale de ce mois-ci (numéro 3, juin 2020) décrivent certaines des études présentées au congrès de la SCEB tenu à l'Université Carleton à Ottawa (Ontario) du 13 au 15 mai 2019 sous le thème de " L'épidémiologie et la biostatistique au cœur des défis complexes en matière de santé ». Les participants ont été invités à soumettre des manuscrits adaptés des travaux qu'ils ont présentés à ce congrès. Les manuscrits ont ensuite été assujettis au mécanisme habituel d'évaluation par les pairs de la RCSP.

Les six articles publiés dans la rubrique spéciale portent sur un vaste éventail de sujets et couvrent une importante sélection de la recherche épidémiologique en cours au Canada. Ils abordent des questions méthodologiques, des recherches étiologiques sur les nourrissons et les populations vieillissantes, les déterminants sociaux de la santé et des analyses de données administratives sur la santé.

Bauer et collègues (Bauer et al., 2020) expriment d'importantes réserves quant au langage actuellement utilisé dans les enquêtes canadiennes pour classifier les minorités visibles et montrent que les questions de ces enquêtes ne saisissent pas de façon fiable les personnes qui sont racisées en tant que personnes de couleur. Les auteurs cherchent à comprendre comment des Canadiens et Canadiennes d'antécédents divers interprètent les expressions «personne de couleur » et " minorité visible », et ils formulent des recommandations pour que les enquêtes futures puissent mieux saisir la racisation. Ce travail fournit des preuves empiriques qui aideront les chercheurs à intégrer des considérations d'équité, de diversité et d'inclusivité dans leurs études.

Duchaine et collègues (Duchaine et al., 2020) offrent une élégante analyse des données de l'Étude longitudinale canadienne sur le vieillissement en explorant les associations 
entre la vitamine $\mathrm{D}$ et l'apparition de la démence. L'étude fait appel à des mesures plasmatiques de la vitamine $\mathrm{D}$ provenant de certains participants de la cohorte prospective. Les résultats de l'étude confirment l'importance d'examiner les associations différentielles entre les hommes et les femmes. Les auteurs indiquent le besoin de recherches additionnelles sur ces associations, particulièrement chez les femmes, en tenant compte du biais de survie.

À l'autre extrémité du spectre de l'âge, $H u$ et collègues $(\mathrm{Hu}$ et al., 2020) examinent les associations entre les concentrations prénatales des métabolites urinaires des phtalates et les naissances avant terme. Leurs analyses ont été effectuées à l'aide des données de près de 2000 participantes de l'Étude mère-enfant sur les composés chimiques de l'environnement (MIREC), dont les concentrations de métabolites urinaires des phtalates ont été mesurées au cours du premier trimestre de grossesse. Bien qu'en général aucune association entre l'exposition aux phtalates et les naissances avant terme n'ait été trouvée, les tendances non linéaires ont montré des signes de relations dose-réponses non monotones, particulièrement chez les nourrissons de sexe masculin. Selon les auteurs, il faudrait pousser la recherche en utilisant plusieurs indicateurs de phtalates.

L'article de Norwood et collègues (Norwood et al., 2020) cherche à estimer et à cartographier des indicateurs par petite région des déterminants sociaux de la santé à l'aide d'un modèle bayésien qui tient compte des corrélations spatiales des données et qui en mesure l'incertitude. Cette approche surmonte certaines contraintes de l'analyse factorielle, qui est encore la méthode la plus couramment utilisée pour créer des indicateurs canadiens de marginalisation. Les auteurs proposent d'approfondir leur approche en incluant des modèles bayésiens à niveaux multiples intégrant à la fois des données régionales individuelles et contextuelles.

L'étude de Rosella et collègues (Rosella et al., 2020) estime, sur une base régionale, la contribution du statut d'immigration aux variations de la mortalité prématurée en Ontario entre 1992 et 2012. Les résultats indiquent que « l'effet de l'immigrant en bonne santé » contribue beaucoup à la variabilité spatiale de la mortalité. Des démarches pour définir cet effet à une plus fine résolution spatiale représentent une piste de recherche importante, tout comme la capacité de distinguer les familles en utilisant des catégories restreintes en fonction de la date d'établissement.

Sandhu et collègues (Sandhu et al., 2020) présentent une revue secondaire d'une série d'études d'intervention non randomisées visant à prévenir l'obésité chez les enfants et les jeunes. Bien que les interventions examinées soient très prometteuses, la plupart ne tiennent pas suffisamment compte de l'agrégation, n'appliquent pas les méthodes causales avancées utilisées en épidémiologie et, en général, n'évaluent pas les interactions statistiques. Ces études originales présentent donc probablement des indicateurs d'associations biaisés ou surévaluent la précision de leurs indicateurs d'associations.

Dans leur ensemble, ces articles illustrent le dynamisme de la recherche épidémiologique au Canada et ses importantes contributions à l'amélioration de notre compréhension de la santé publique. Ils soulignent aussi l'importance d'employer des méthodes rigoureuses et d'appliquer des principes épidémiologiques solides pour concevoir des études populationnelles et analyser des données de santé publique. Enfin, ils nous rappellent que les collaborations et les partenariats étroits sont essentiels entre les communautés canadiennes de l'épidémiologie, de la biostatistique et de la santé publique, pour l'amélioration de la santé et du bien-être de nos concitoyens.

Paul Villeneuve, Gilles Paradis, Nazeem Muhajarine

Co-rédacteurs, Section spéciale sur l'épidémiologie et la biostatistique

\section{References/Références bibliographiques}

Bauer, G. R., Mahendran, M., Braimoh, J., Alam, S., \& Churchill, S. (2020). Identifying visible minorities or racialized persons on surveys: can we just ask? Can J Public Health 111. https://doi.org/10. 17269/s41997-020-00325-2.

Duchaine, S. C., Talbot, D., Nafti, M., Giguère, Y., Dodin, S., Tourigny, A., Carmichael, P.-H., \& Laurin, D. (2020). Vitamin D status, cognitive decline and incident dementia: the Canadian Study of Health and Aging. Can J Public Health 111. https://doi.org/10.17269/ s41997-019-00290-5.

Hu, J.M.Y., Arbuckle, T.E., Janssen, P., Lanphear, B.P., Braun, J.M., Robert W. Platt, R.W., Chen, A., Fraser, W.D., McCandless, L.C. (2020.) Associations of prenatal urinary phthalate exposure with preterm birth: the Maternal-Infant Research on Environmental Chemicals (MIREC) Study. Can J Public Health 111. https://doi. org/10.17269/s41997-020-00322-5

Norwood, T. A., Encisa, C., Wang, X., Seliske, L., Cunningham, J., \& De, P. (2020). A Bayesian shared components modeling approach to develop small area indicators of social determinants of health with measures of uncertainty. Can J Public Health 111. https://doi.org/ 10.17269/s41997-020-00321-6.

Rosella, L. C., Kornas, K., Watson, T., Buajitti, E., Bornbaum, C., Henry, D., \& Brown, A. (2020). Association between the regional variation in premature mortality and immigration in Ontario, Canada. Can J Public Health 111. https://doi.org/10.17269/s41997-020-00330-5.

Sandhu, R., Mbuagbaw, L., Tarride, J.-E., De Rubeis, V., Carsley, S., \& Anderson, L. N. (2020). Methodological approaches to the design and analysis of nonrandomized intervention studies for the prevention of child and adolescent obesity. Canadian Journal of Public Health, 111 .

Publisher's note Springer Nature remains neutral with regard to jurisdictional claims in published maps and institutional affiliations. 